
zonafranca
eGon:
CENTRO DE ESTUDIOS
INTERDISCIPLINARIOS SOBRE MUJERES
MU MAESTRIA "PODER Y SOCIEDAD

\title{
Ley Micaela en la Universidad Nacional de Lanús \\ Aportes para la erradicación de la violencia por razones de género
}

\author{
Andrea Vallejos \\ Victoria Freire* *
}

\section{Resumen}

El presente artículo se propone en primer término contextualizar la aprobación de la capacitación obligatoria como parte de un proceso de visibilización de las violencias por razones de género a nivel social y en el sistema universitario, y su vínculo con una agenda integral de políticas para la erradicación de las violencias y la promoción de la igualdad de género en el ámbito universitario a partir del caso de la Universidad Nacional de Lanús. En el segundo apartado se comparten las características de la capacitación brindada, y finalmente se presenta la información específica del estado de implementación de la Ley a diciembre de 2020 en la Universidad Nacional de Lanús, en particular el análisis de la encuesta que se implementa al finalizar el Curso dirigido a trabajadoras y trabajadores no docentes.

Palabras clave: violencia, capacitación, universidad

\footnotetext{
*Andrea Vallejos, es trabajadora social (UNLa), especialista en Políticas Públicas y justicia de Género (CLACSO/ FLACSO BRASIL) coordinadora del Programa por la Igualdad de Género de la Universidad Nacional de Lanús. Contacto: vallejos.ab@gmail.com

* * Victoria Freire, es socióloga (UBA), directora del Observatorio de Género y Políticas Públicas e integrante de la Fundación Igualdad. Contacto: Victoriafreire85@gmail.com
}

Vallejos, Andrea; Freire, Victoria. "Ley Micaela en la Universidad Nacional de Lanús. Aportes para la erradicación de la violencia por razones de género" en Zona Franca. Revista del Centro de estudios Interdisciplinario sobre las Mujeres, y de la Maestría poder y sociedad desde la problemática de Género, №29, 2021 pp. 353-370. ISSN, 2545-6504 Recibido: 27 de abril 2021; Aceptado: 15 de noviembre 2021.

Revista Zona Franca- Centro de estudios interdisciplinario sobre las mujeres (CEIM)- Maestría poder y sociedad desde la problemática de género (MG), Rosario, Argentina. ISSN, 2545-6504 http://zonafranca.unr.edu.ar/index.php/ZonaFranca| Número 29 (2021). 


\title{
Contributions for the eradication of gender-based violence at the National University of Lanús
}

\begin{abstract}
This article aims to first contextualize the approval of compulsory training as part of a process of making violence visible at the social level and in the university system, and its link with a comprehensive policy agenda for the eradication of violence and the promotion of gender equality at the university level based on the case of the National University of Lanús. In the second section, the characteristics of the training provided are shared and finally the specific information on the state of implementation of the Law as of December 2020 at the National University of Lanús is presented and in particular an analysis of the survey that is implemented at the end of the Course aimed at non-teaching workers.
\end{abstract}

Keywords: violence, gender, higher education

\section{Presentación}

El 19 de diciembre de 2018 se aprobó en la Argentina la Ley № 27.499 que lleva el nombre Micaela García, víctima de un brutal femicidio, "en reconocimiento por su lucha militante por los derechos de las mujeres y de los más vulnerables"'.

La ley tiene por objeto la "capacitación obligatoria en la temática de género y violencia contra las mujeres para todas las personas que se desempeñen en la función pública en todos sus niveles y jerarquías en los poderes Ejecutivo, Legislativo y Judicial de la Nación"”l.

Un día después de la aprobación de la Ley, la Universidad Nacional de Lanús decidió fortalecer su compromiso con la igualdad de género y la erradicación de las violencias por razones de género a partir de la aprobación con carácter de obligatorio de la capacitación en género y diversidad con alcance a personal

I Fundamentos del Proyecto de Ley.

"Ley 27499, artículo 1. Norma completa disponible en: http://servicios.infoleg.gob.ar/infoleglnternet/anexos/315000-319999/318666/norma.htm

Revista Zona Franca- Centro de estudios interdisciplinario sobre las mujeres (CEIM)- Maestría poder y sociedad desde la problemática de género (MG), Rosario, Argentina. ISSN, 2545-6504 http://zonafranca.unr.edu.ar/index.php/ZonaFrancal Número 29 (2021). 
superior, docentes y nodocentes de la Universidad, por medio de la resolución de Consejo Superior $\mathrm{N}^{\circ} 239 / 18^{\prime \prime \prime}$.

A partir de las articulaciones institucionales llevadas adelante entre áreas encargadas de las capacitaciones destinadas a docentes y no docentes de la Universidad, y las asociaciones sindicales de base (Asociación de Trabajadores y Trabajadoras de la Universidad Nacional de Lanús y Asociación de Docentes Investigadores de la Universidad Nacional de Lanús) en el año 2019 comenzó la implementación de distintos espacios de formación:

- El Curso "Géneros, identidades y educación superior" destinado a docentes se implementa en el marco del Programa de Capacitación Docente Continua (PROCADO) $)^{\mathrm{IV}}$.

- El Curso "Género y diversidad"V para la formación de las y los no docentes y del personal superior ${ }^{\mathrm{VI}}$ desarrollado desde el Programa por la Igualdad de Género dependiente de la Secretaría de Bienestar y Compromiso Universitario.

Es interesante destacar que estas propuestas surgen de las mesas interclaustros que se convocaron desde la Universidad para trabajar estrategias vinculadas a la participación y desarrollo de los paros feministas en el marco de la comunidad universitaria.

III Disponible en: http://www.unla.edu.ar/resoluciones/2018/Diciembre/R.CS.N 239-18-20.12.18\%20Incorporar\%20Curso\%200bligatorio\%20Genero\%20y\%20Diversidad\%20para\%20PS \%20ND\%20Doc.pdf

IV Mas información sobre el Programa PROCADO en: http://www.unla.edu.ar/secretarias/academica/direccion-de-pedagogia-universitaria/procadoprograma-de-capacitacion-docente

v Mas información en: http://www.unla.edu.ar/resoluciones/2018/Diciembre/R.CS.N 239-18-20.12.18\%20Incorporar\%20Curso\%200bligatorio\%20Genero\%20y\%20Diversidad\%20para\%20PS $\% 20$ ND\%20Doc.pdf

${ }^{\mathrm{VI}}$ Rectora, Vicerrector, Secretarios y Secretarias y Directores de Departamento.

Revista Zona Franca- Centro de estudios interdisciplinario sobre las mujeres (CEIM)- Maestría poder y sociedad desde la problemática de género (MG), Rosario, Argentina. ISSN, 2545-6504 http://zonafranca.unr.edu.ar/index.php/ZonaFrancal Número 29 (2021). 
Por su parte el Consejo Interuniversitario Nacional (CIN) en su plenario realizado el 8 de abril de 2018, recomendó que el sistema universitario adhiera de la Ley N²7.499 "LEY MICAELA"VII.

El presente artículo se propone en primer término contextualizar la aprobación de la capacitación obligatoria como parte de un proceso de visibilización de las violencias a nivel social y en el sistema universitario, y su vínculo con una agenda integral de políticas para la erradicación de las violencias y la promoción de la igualdad de género en el ámbito universitario a partir del caso de la Universidad Nacional de Lanús. En el segundo apartado se comparten las características de la capacitación brindada, y finalmente se presenta la información específica del estado de implementación de la Ley a diciembre de 2019 en la Universidad Nacional de Lanús, en particular el análisis de la encuesta que se implementa al finalizar el Curso dirigido a trabajadoras y trabajadores no docentes.

Cabe señalar que el inicio del Plan de capacitación es mayo de 2019, destinado en primera instancia al personal superior, Rectora, Vice rector, Secretarios y Secretarías y Directores de Departamento. El mismo se dictó desde la Secretaría de Bienestar y Compromiso Universitario, y las docentes del curso fueron Andrea Vallejos junto a Victoria Freire.

\section{Sobre las políticas de género y contra las violencias en las} universidades

La primer convocatoria en 2015 de \#NiUnaMenos en Argentina ha significado un momento de inflexión tanto para los feminismos como en la sociedad, caracterizado por la sensibilización acerca de las formas más extremas de la violencia machista ante la alarmante cifra de femicidios.

VII Registro de la noticia disponible en: https://www.argentina.gob.ar/noticias/el-sistema-universitarioadherira-la-ley-micaela

Revista Zona Franca- Centro de estudios interdisciplinario sobre las mujeres (CEIM)- Maestría poder y sociedad desde la problemática de género (MG), Rosario, Argentina. ISSN, 2545-6504 http://zonafranca.unr.edu.ar/index.php/ZonaFranca| Número 29 (2021). 
En este sentido cabe señalar como expresa Arzamendia (2017) que:

"Si bien es cierto el colectivo nació en reclamo a los femicidios, pronto fue ampliando sus demandas, en tanto que se reconocían otras formas de opresión y violencia de género. Se expresaba que el femicidio es la expresión más extrema de una serie mucha más larga y numerosas veces invisible de violencia hacia la mujer". (2017: 2)

Feministas argentinas sostienen que a partir de dicho momento la conciencia colectiva acerca de las violencias se amplió, aunque éstas no disminuyeron VIII.

La violencia por razones de género ha sido uno de los temas más denunciados por el movimiento feminista. Particularmente en Latinoamérica, este movimiento es, en palabras de Alba Carosio (2012), "un movimiento de descontento con lo 'cotidiano' y respecto al 'así son las cosas', que saca a la luz la dominación y las relaciones de poder en lo personal y privado, en las que se funda la dominación social" (2012: 242).

Al descontento y el deseo de transformar la realidad, que en el año 2015 reflejaba que se producía en la Argentina un femicidio cada 30 horas, se le sumó la indignación ante el caso de Chiara Páez, una adolescente de 14 años oriunda de Rufino en la provincia de Santa Fe, que fue asesinada a golpes por su novio el domingo 10 de mayo de 2015. El dolor se tradujo en organización y en un grito colectivo que desde ese año colma las calles con el reclamo de \#NiUnaMenos, \#VivasNosQueremos.

Además de los reclamos concretos del movimiento, se produjo una profundización de la comprensión y visibilidad de las violencias, así como muchas personas que a partir de entonces se sumaron a este movimiento. Diferentes acciones colectivas comenzaron a desarrollarse en diversos ámbitos durante los

VIII Ver: https://latfem.org/a-cuatro-anos-de-ni-una-menos-avances-y-retrocesos-a-nivel-nacionalcaba-cordoba-y-rosario/

Revista Zona Franca- Centro de estudios interdisciplinario sobre las mujeres (CEIM)- Maestría poder y sociedad desde la problemática de género (MG), Rosario, Argentina. ISSN, 2545-6504 http://zonafranca.unr.edu.ar/index.php/ZonaFranca| Número 29 (2021). 
siguientes años. Asimismo, hubo un impacto amplio y diverso en las agendas de las instituciones, incluidas las Universidades.

En este contexto en las Universidades nacionales también se dan procesos de problematización de situaciones históricamente naturalizadas. Surgen investigaciones que dan cuenta de las distintas formas de violencias vinculadas a los géneros, sobre los comentarios sexistas y el acoso, que toman relevancia en dichos estudios $^{\mathrm{IX}}$. Universidades de distintos puntos del país comienzan a gestar espacios y procedimientos que permiten la actuación ante situaciones de violencia que involucran personas que integran la comunidad universitaria. En 2013 la Universidad Nacional De Lanús ${ }^{\mathrm{X}}$ y la Universidad Nacional de San Martín ${ }^{\mathrm{XI}}$ crean programas que brindan espacios de escucha y acompañamiento ante situaciones de violencia. En 2015 la Universidad de Comahue aprueba un protocoloXII.

En 2015 toma fuerza en el sistema universitario un entramado de articulaciones que se materializa en la Red por la Igualdad de género y contra las violencias. A partir de este momento surgen diversas articulaciones y experiencias en las universidades principalmente vinculadas a la prevención, intervención y erradicación de las violencias, aunque no únicamente, como señala Moltoni (2018) "los cuestionamientos al orden androcéntrico universitario emergieron con más fuerza al calor de las reivindicaciones del movimiento feminista, impulsando

Ix En el año 2011 Maite Rodigou Nocetti, Paola Blanes, Jacinta Burijovich y Alejandra Domínguez presentaron el libro Trabajar en la Universidad. (Des)igualdades de Género por transformar. En el año 2011 se llevó adelante en la misma Universidad el estudio "Violencia de Género en estudiantes universitarios", desde la carrera de Trabajo Social de la Facultad de Ciencias Sociales y el área de Feminismos, Géneros y

Sexualidades del Centro de Investigaciones - Facultad de Filosofía y Humanidades. en el año 2016 la Universidad Nacional de San Martin llevó adelante el estudio denominado "Diagnostico sobre discriminación y violencia".

x Universidad Nacional de Lanús. Resolución de Consejo Superior № 159/13 del 16/08/2013. Consultada en: http://www.unla.edu.ar/resoluciones/2013/Agosto/R.CS.N 159-13-16.08.13\%20Res.Programa\%20por\%20la\%20lgualdad\%20de\%20G\%C3\%A9nero.pdf

XI Fuente: Página 12, Suplemento Las12. Protocolo contra violencia de género. Por Vanesa Vázquez Laba, Coordinadora del Programa contra la Violencia de Género de la Universidad Nacional de San Martín. Viernes 4 de diciembre de 2015. Disponible en:

https://www.pagina12.com.ar/diario/suplementos/las12/13-10225-2015-12-04.html

XII Disponible en https://ranquel.uncoma.edu.ar/archivos//ord 15722014 23.pdf.

Revista Zona Franca- Centro de estudios interdisciplinario sobre las mujeres (CEIM)- Maestría poder y sociedad desde la problemática de género (MG), Rosario, Argentina. ISSN, 2545-6504 http://zonafranca.unr.edu.ar/index.php/ZonaFranca| Número 29 (2021).

Página 358 
acciones concretas en pos de la prevención, atención y erradicación de las violencias de género" (2018: 54).

En el 2018 la Red se incorpora como organización en el marco del Consejo Interuniversitario Nacional (CIN), desde donde se propone colaborar en el diseño y desarrollo de políticas que contribuyan a erradicar las desigualdades de género y las violencias en todo el sistema universitario. Con las siglas "RUGE" como nombre abreviado, se designan las autoridades para el primer período 2018-2020, quedando como coordinadora Sandra Torlucci, Rectora de la Universidad Nacional de las Artes ${ }^{\mathrm{XIII}}$.

Desde la RED RUGE se proponen diversos ejes de trabajo y estrategias vinculadas a la agenda de género en las universidades. Una de las estrategias fue la adhesión a la Ley Micaela. Torlucci y Vazquez Laba y Pérez Tort, refieren al respecto que:

"La presentación del proyecto de adhesión a la "Ley Micaela" en el plenario de Rectores del Consejo Interuniversitario Nacional en el mes de mayo, fue otro paso decisivo de la acción colectiva y estratégica feminista. Presentado por la Rectora coordinadora de la RUGE, Sandra Torlucci y acompañado por las palabras de "Yuyo" García, el papá de Micaela, el proyecto fue aprobado por unanimidad". (2019: 7)

Según un relevamiento realizado por la RED RUGE ${ }^{X I V}$ en el año 2019 el 81,97 por ciento de las universidades han adherido a la Ley Micaela mientras que el 49 por ciento ya habían comenzado con la implementación de las capacitaciones.

\section{El recorrido de la Universidad Nacional de Lanús}

XIII Extraído de página web de la Red: http://ruge.cin.edu.ar/informacion/historia

XIV Los datos del relevamiento pueden consultarse en: http://ruge.cin.edu.ar/attachments/article/43/RUGE.\%20Relevamiento\%20principales\%20resultado $\underline{\mathrm{s} \% 200702 \% 20(4) . p d f}$

Revista Zona Franca- Centro de estudios interdisciplinario sobre las mujeres (CEIM)- Maestría poder y sociedad desde la problemática de género (MG), Rosario, Argentina. ISSN, 2545-6504 http://zonafranca.unr.edu.ar/index.php/ZonaFrancal Número 29 (2021). 
La UNLa fue creada por medio de la Ley № 24.496, sancionada el 7 de junio de 1995, en el distrito de Lanús en el conurbano bonaerense. Desde su creación, la promoción de la igualdad y la defensa de los derechos humanos forman parte de su finalidad y principios que se fueron fortaleciendo a partir del desarrollo de políticas de investigación, cooperación, formación e intervención institucional. En pocos años se aprobaron programas institucionales $\mathrm{Xv}$, se incorporaron materias optativas $\mathrm{y}$ contenidos transversales y obligatorio sobre cuestiones de género y derechos humanos $^{\mathrm{XVI}}$, se aprobó una carrera de posgrado ${ }^{\mathrm{XVII}}$ y se aprobaron procedimientos internos para intervenir ante situaciones de violencias ${ }^{\mathrm{XIII}}$.

En ese marco, la articulación de la agenda de género de la universidad con las movilizaciones masivas que convocaron al encuentro y la reflexión sobre cómo se reproducen las desigualdades de género, posibilitaron también al interior de la universidad la coordinación de diversos actores gremiales e institucionales con el objetivo de promover acciones para el fortalecimiento de la igualdad y la prevención de la violencia.

Al calor de las movilizaciones y paros feministas en el año 2017 se realizaron encuentros inter claustros que favorecieron la construcción de propuestas vinculadas a la capacitación de trabajadores y trabajadoras de la universidad. Desde ese momento se trabajó en torno a dos proyectos que si bien se presentaron para

\footnotetext{
xv En 2008 se crea el Programa Género y Políticas Públicas y en 2013 el Programa por la Igualdad de Género: Resolución de Consejo Superior № 159/13 del 16/08/2013. Consultada en: http://www.unla.edu.ar/resoluciones/2013/Agosto/R.CS.N 159-13-16.08.13\%20Res.Programa\%20por\%20la\%20lgualdad\%20de\%20G\%C3\%A9nero.pdf

XVI En el año 2013 la UNLa por Resolución del Consejo Superior № 231/13, la UNLa estableció como requisito para todos los ingresantes de las carreras de pre grado y grado, el Seminario de Justicia y Derechos Humanos, que incorpora contenido vinculados a género y derechos humanos.

XVII En el año 2014 se aprobó la especialización en Género, Políticas Públicas y Sociedad. Más información en: http://www.unla.edu.ar/carreras/posgrado/especializaciones/genero-politicaspublicas-y-sociedad

XVIII En el año 2017 se aprobó por medio de la R.C.S. N 200/17 el Protocolo de intervención ante situaciones de violencia por género, orientación sexual, identidad y/o expresión de género. Disponible en: http://www.unla.edu.ar/resoluciones/2017/Diciembre/R.CS.N 200-17-13.12.17\%20Aprobar\%20Protocolo\%20intervencion\%20ante\%20situaciones\%20discriminacion $\% 2$ 0o\%20violencia\%20genero.pdf
}

Revista Zona Franca- Centro de estudios interdisciplinario sobre las mujeres (CEIM)- Maestría poder y sociedad desde la problemática de género (MG), Rosario, Argentina. ISSN, 2545-6504 http://zonafranca.unr.edu.ar/index.php/ZonaFrancal Número 29 (2021). 
tratamiento del Consejo Superior en carácter optativo, la aprobación finalmente fue de carácter obligatorio.

En diciembre de 2018 el Consejo Superior de la Universidad trataba un proyecto que proponía la capacitación optativa destinada al personal no docente de la Universidad, elevado por el Programa por la Igualdad de Género en articulación con el Comité Técnico Mixto de Capacitación y Formación Profesional. En dicha reunión se decidió que dicha propuesta debía adquirir carácter obligatorio y hacerse extensivo a docentes y personal jerárquico. Esta decisión institucional es producto de varias cuestiones, sin embargo interesa destacar que se produce en los últimos años de avance y creciente interpelación de los discursos feministas que han permitido la ampliación de la conciencia colectiva acerca de la problemática de las violencias contra las mujeres y LGBTIQ, que se articula con una mayor problematización de las formas de violencia que se producen en los ámbitos universitarios así como del rol que deben asumir dichas instituciones para prevenir, intervenir y erradicar sus manifestaciones. Este proceso que se desarrolla en las universidades asume características particulares en la UNLa vinculadas al proyecto institucional, los valores y fundamentos de la universidad.

\section{El itinerario formativo sobre género y diversidad para trabajadoras}

\section{y trabajadores}

En el mes de mayo de 2019 comenzó el primer dictado de la capacitación destinado a las autoridades que conforman el gabinete de la Universidad, desde la Secretaría de Bienestar y Compromiso Universitario, a través del equipo del Programa por la Igualdad de Género.

En junio del mismo año comenzaron las capacitaciones destinadas al personal no docente, que se dictaron en dos momentos: el primero de modalidad presencial y el segundo de carácter virtual, con dos encuentros presenciales.

Revista Zona Franca- Centro de estudios interdisciplinario sobre las mujeres (CEIM)- Maestría poder y sociedad desde la problemática de género (MG), Rosario, Argentina. ISSN, 2545-6504 http://zonafranca.unr.edu.ar/index.php/ZonaFranca| Número 29 (2021). 
El Programa del Curso ofrece un itinerario que recorre desde la primera unidad conceptos nodales de la teoría de género y feminista vinculando los mismos a los contextos socios históricos de surgimiento, las luchas feministas por la igualdad, las conquistas en términos de derechos, las herramientas de abordaje de las violencias de género y el desarrollo de políticas públicas.

Para dar inicio al curso recuperamos a Micaela García, joven militante que fue víctima de un femicidio en Entre Ríos. La ley que lleva su nombre tiene como objetivo "capacitar y sensibilizar a quienes integran los diferentes estamentos del Estado" entendido no como una mera elección de preferencia personal sino a los fines de dar cumplimiento a un deber que asumió nuestro país al aprobar la Convención Interamericana para prevenir, sancionar y erradicar la violencia contra la mujer, "Belém do Pará".

A partir de esta contextualización invitamos a pensar nuestras prácticas institucionales, nuestra vida cotidiana y la política institucional, haciendo una conexión entre la formación en igualdad y la formación para la igualdad. Desde esta perspectiva entendemos que la educación constituye una práctica transformadora (Rodino, 2014) que favorece la transformación de nuestras propias experiencias y acciones, así como las prácticas y política institucional.

Analizamos las principales demandas y conquistas del movimiento feminista en los distintos contextos socios históricos, construimos una línea de tiempo que se propone ser enriquecida colectivamente. Propiciamos la identificación específica de las demandas feministas en Latinoamérica y Argentina. Analizamos la emergencia de los conceptos de género, patriarcado e interseccionalidad y sus implicancias para la comprensión de las desigualdades entre los géneros.

En la segunda unidad se abordan las conceptualizaciones respecto de la diversidad sexual y las identidades de género. Trabajamos los conceptos de heteronormatividad y binarismos de género, para reflexionar sobre la construcción de las diversas identidades sexo genéricas. Trabajamos en torno al marco

Revista Zona Franca- Centro de estudios interdisciplinario sobre las mujeres (CEIM)- Maestría poder y sociedad desde la problemática de género (MG), Rosario, Argentina. ISSN, 2545-6504 http://zonafranca.unr.edu.ar/index.php/ZonaFranca| Número 29 (2021). 
normativo de derechos de las personas del colectivo LGBTI+. Abordamos específicamente la Ley de identidad de género de Argentina, las disposiciones internas de la Universidad respecto de la misma y los procedimientos establecidos para el cumplimiento de lo estipulado en ellas.

En el tercer momento dentro de la planificación del curso, en la unidad 3, nos enfocamos en las diferentes formas de violencias vinculadas al género, la orientación sexual y la identidad de género. Para esto comenzamos problematizando las formas de violencias que conocemos $\mathrm{y} / \mathrm{o}$ identificamos, para luego abordar las formas naturalizadas. Partimos de las experiencias puestas en común en el curso respecto a los tipos y las modalidades de las violencias para luego vincularlas con la definiciones y alcances que establece la Ley 26.485 de Protección Integral para prevenir, sancionar y erradicar las violencias contra las mujeres en todos los ámbitos en que desarrollan sus relaciones interpersonales. En torno a estas violencias reconstruimos colectivamente las diversas estrategias que se desarrollan, desde las redes comunitarias así como las políticas públicas destinadas a su atención y abordaje. Situándonos en el marco de la Universidad desarrollamos el protocolo de intervención ante situaciones de violencia y/ o discriminación aprobado por el Consejo Superior en diciembre de 2017. Con el objetivo de contextualizar estos procesos en el sistema universitario, se analizan los datos en torno a la aprobación de protocolos en todo el país realizado por la Red RUGE, socializamos los objetivos y ejes de trabajo de dicha red.

A partir del intercambio en torno a la herramienta del protocolo proponemos pensar en las situaciones que pueden presentarse y reflexionar colectivamente sobre las estrategias que podemos implementar como integrantes de la Universidad para atender y erradicar estas formas de violencia.

Por último, en la unidad 4 del curso propusimos repensar algunos ejes desde la transversalidad de la perspectiva de género, articulando los conceptos trabajados 
hasta el momento. En este tramo se pretende principalmente recuperar las inquietudes específicas de las y los participantes respecto de la temática.

\section{Datos sobre la implementación de la capacitación}

A continuación se presentan los principales datos acerca de la implementación de la capacitación desde su inicio.

En la primera etapa de implementación del curso se inscribieron 161 trabajadores y trabajadoras nodocentes. Participaron y aprobaron el curso 112 personas, es decir el 70 por ciento de quienes se inscribieron lo finalizaron.

Durante el año 2019 la capacitación se dictó en dos modalidades, presencial $y$ en dos comisiones virtuales ${ }^{X I X}$ a través de la plataforma del Campus virtual de la UNLa. La participación en ambas instancias fue concurrida.

Durante el año 2020 con el contexto de pandemia y el Aislamiento Social, Preventivo y Obligatorio se debió implementar la totalidad de la oferta de manera virtual, notando una mayor concurrencia durante el primer cuatrimestre. Del Total de la Planta No docente de la Universidad en el año 2020, que es de 371 trabajadores y trabajadoras ${ }^{\mathrm{Xx}}$, han participado del curso el 45 por ciento. Compartimos a continuación un material de difusión elaborado por la universidad.

XIX En el caso de la instancia virtual, constó de 6 encuentros virtuales y dos encuentros presenciales. xx Corresponde a Nodocentes con cargos activos. Información recabada del Informe de Gestión UNLa 2020. Página 162. Informe completo disponible en: http://www.unla.edu.ar/documentos/Agenda\%20Estad\%C3\%ADstica\%202020.pdf

Revista Zona Franca- Centro de estudios interdisciplinario sobre las mujeres (CEIM)- Maestría poder y sociedad desde la problemática de género (MG), Rosario, Argentina. ISSN, 2545-6504 http://zonafranca.unr.edu.ar/index.php/ZonaFranca| Número 29 (2021). 


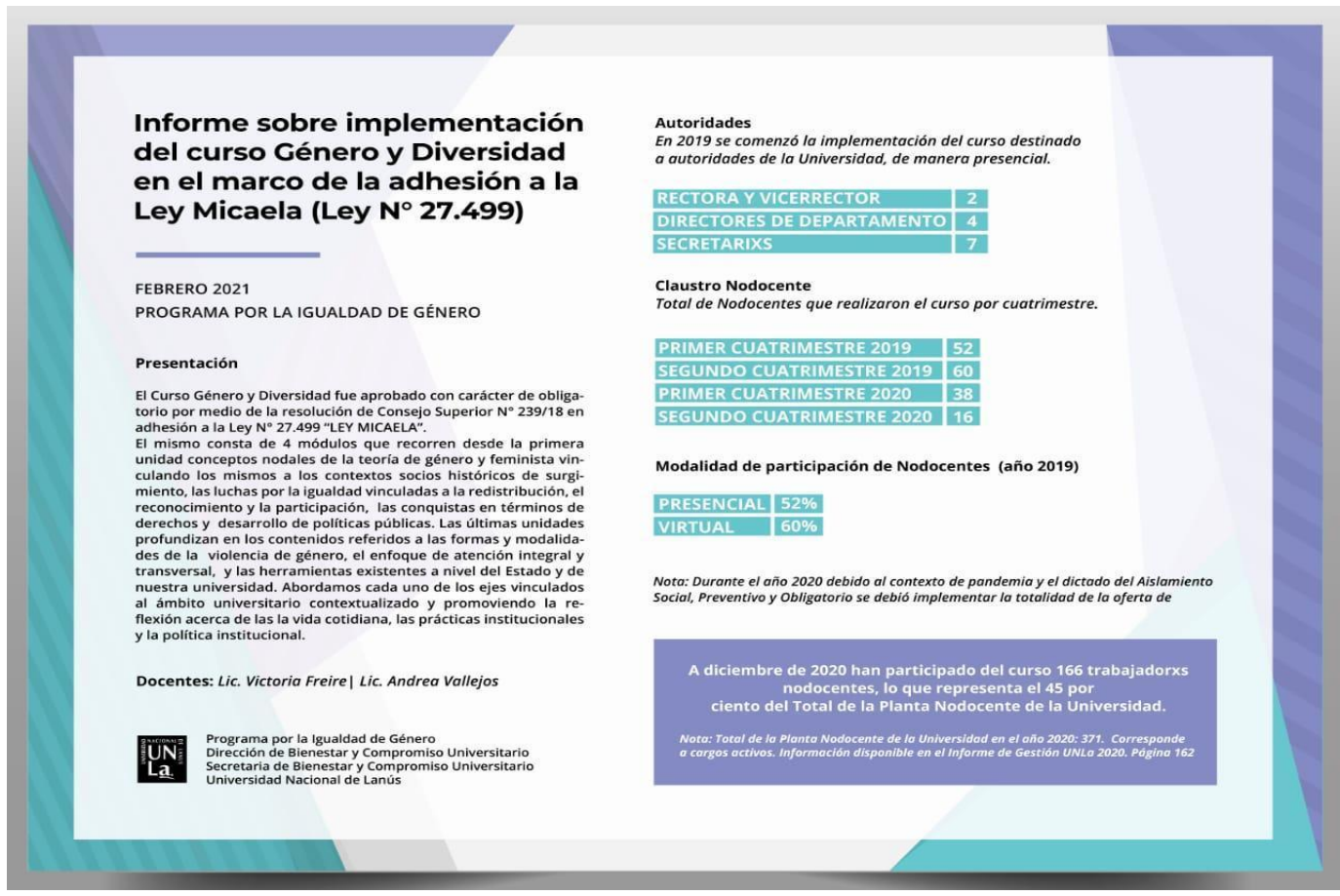

Para finalizar el itinerario formativo se comparten dos consignas para un trabajo final integrador sobre la actuación del protocolo en la universidad y propuestas competentes al área de trabajo, que son evaluadas con comentarios particulares en cada caso, y una encuesta para responder individualmente sobre las características del curso. En el siguiente apartado compartimos los principales hallazgos surgidos de dicha encuesta.

\section{Algunas reflexiones para compartir}

La encuesta de finalización es carácter optativo y anónimoXXI. Esta encuesta se desarrolla como instancia de retroalimentación para conocer algunos aspectos que consideramos principales para enriquecer los futuros procesos de formación.

Mediante esta encuesta se indagan, entre otros ejes, si el curso favoreció a aclarar, profundizar o modificar las percepciones previas sobre géneros y

XXI La encuesta se aplicó en las tres últimas comisiones del curso: incluye segundo cuatrimestre de 2019, primer y segundo cuatrimestre de 2020. Respondieron a la misma 70 trabajadorxs nodocentes.

Revista Zona Franca- Centro de estudios interdisciplinario sobre las mujeres (CEIM)- Maestría poder y sociedad desde la problemática de género (MG), Rosario, Argentina. ISSN, 2545-6504 http://zonafranca.unr.edu.ar/index.php/ZonaFranca| Número 29 (2021). 
diversidad. Asimismo se consulta respecto de algunos ejes relacionados con el funcionamiento del curso: contenidos, docentes, dinámicas, entre otros.

Sobre la pregunta ¿Qué relación encontrás entre tu inserción laboral/ institucional y las cuestiones de género y diversidad vista en el curso? El 86 por ciento responde que existe una vinculación, para el 60 por ciento es una relación directa, mientras que el 26 por ciento encuentra una relación indirecta. Solo el 14 por ciento señala que no existe ninguna relación.

Para evaluar el impacto de los contenidos en diferentes ámbitos, desde lo institucional hasta las cuestiones ligadas a la vida cotidiana, se propusieron ocho frases para ser contestadas según el nivel de acuerdo. Se obtuvieron las siguientes respuestas:

- El 78.5 $\mathrm{XXI1}$ por ciento refirió estar de acuerdo con la afirmación que plantea que los contenidos del curso ayudaron a identificar temas y problemas que se relacionan con la tarea cotidiana en la Universidad.

- Respecto a si el curso aportó a pensar en los derechos de las personas con las que se vinculan en el lugar de trabajo (compañerxs y estudiantes), el 76 por ciento estuvo de acuerdo.

- Sobre si el curso aportó a la reflexión es aspecto de la vida cotidiana, no solo en la universidad, el 51 por ciento de quienes participaron del curso señalaron estar muy de acuerdo, mientras que el 20 por ciento señalaron estar algo de acuerdo.

- Respecto a si el curso brindó una nueva perspectiva para entender la realidad, recuperando los objetivos de incorporar la perspectiva de género como una lente para comprender la realidad, el 54 por ciento estuvo muy de acuerdo y el 31 por ciento estuvo algo de acuerdo.

XXII Se incluyen las respuestas de quienes contestaron algo de acuerdo y muy de acuerdo.

Revista Zona Franca- Centro de estudios interdisciplinario sobre las mujeres (CEIM)- Maestría poder y sociedad desde la problemática de género (MG), Rosario, Argentina. ISSN, 2545-6504 http://zonafranca.unr.edu.ar/index.php/ZonaFrancal Número 29 (2021). 
Por último se indaga acerca de qué piensan respecto a la necesidad de que las universidades cuenten con cursos sobre género y diversidad:

- Con la posibilidad de que sea obligatorio estuvo muy de acuerdo el 73 por ciento, mientras que el 11 manifestó estar algo de acuerdo.

- Respecto a que exista el curso pero que sea de carácter optativo el 37 por ciento estuvo muy de acuerdo mientras que el 23 estuvo de acuerdo.

Estos datos nos permiten conocer el grado de adhesión respecto a lo establecido en la Ley Micaela sobre la capacitación obligatoria en temas de género y violencias. Las personas consultadas adhieren en mayor medida a que esta capacitación sea obligatoria y no optativa.

Las respuestas obtenidas nos muestran la importancia que tiene la formación en torno a estas temáticas no solo por el abordaje y presentación de contenidos teóricos sino también en la apropiación directa de los mismos, la reflexión respecto a las prácticas personales e institucionales, la aplicación en situaciones concretas en la universidad y en otros ámbitos en los que desenvolvemos nuestras vidas cotidianas, la posibilidad de incorporar una nueva perspectiva con la cual observar la realidad, entendiendo las injusticias históricas, contextualizando y reflexionando acerca de cómo se organizan las relaciones de género.

Si bien entendemos que todas las personas que trabajan en la Universidad tienen la obligación (según lo establece la Ley Micaela recuperando las obligaciones del Estado surgida de tratados internacionales) a realizar estas capacitaciones, igualmente es función de la Universidad brindar espacios formativos y en ese sentido toda la comunidad educativa debe asumir las responsabilidades, también creemos que es importante que las personas que transitan este itinerario pedagógico puedan ser interpeladas, que estos espacios les permitan pensar y (re)pensarse, que permita reflexionar, cuestionar y proponer formas situadas,

Revista Zona Franca- Centro de estudios interdisciplinario sobre las mujeres (CEIM)- Maestría poder y sociedad desde la problemática de género (MG), Rosario, Argentina. ISSN, 2545-6504 http://zonafranca.unr.edu.ar/index.php/ZonaFranca| Número 29 (2021). 
colectivas y responsables para la construcción de universidades con mayores grados de igualdad y sin violencias.

Es importante retomar que muchos de los contenidos que recibimos diariamente acerca de estos y otros temas, provienen desde el sentido común, aún cargado de concepciones discriminatorias. Entendiendo esto, decidimos preguntar si el curso había favorecido a modificar las percepciones que tenían sobre las cuestiones de género y diversidad, a lo cual el 76,8 por ciento de quienes transitaron el curso refirieron que sí.

A continuación solicitamos, a modo optativo, que nos cuenten en qué sentido creen que se modificaron sus percepciones. Podemos organizar las respuestas obtenidas en los siguientes ejes:

- Quienes consideran que los conceptos brindados les permiten entender lo cotidiano, tanto personal como laboral.

- Quienes consideran que permitió reformar, actualizar y/o profundizar conocimientos previos.

- Quienes tenían dudas y obtuvieron la posibilidad de preguntar

- Quienes valoran la posibilidad de intercambio y enriquecimiento con otrxs compañerxs.

- Quienes refieren a la posibilidad de reflexionar, sobre las formas de discriminación y violencia

Por último se propuso que puedan expresar lo que les resulte más importante del curso. Si bien se trata de una pregunta abierta, podemos agruparlas de la siguiente manera:

- Es valorado por varias personas el hecho de que el curso brinda información y herramientas concretas ante situaciones de violencia. En ese

Revista Zona Franca- Centro de estudios interdisciplinario sobre las mujeres (CEIM)- Maestría poder y sociedad desde la problemática de género (MG), Rosario, Argentina. ISSN, 2545-6504 http://zonafranca.unr.edu.ar/index.php/ZonaFrancal Número 29 (2021). 
sentido se valora la información respecto de las normativas trabajadas, tanto nacionales (Ley 27.499, ley 26.743, Ley 26.485) como internas (principalmente el protocolo de intervención ante situaciones de violencia y discriminación por género, orientación sexual, identidad y/o expresión de género). También las orientaciones para actuar antes situaciones de violencia y/o discriminación.

- Otro aspecto señalado es la posibilidad de incorporar la perspectiva de género en la vida cotidiana, en el análisis de distintas situaciones que hemos naturalizado. Por último también se valora la forma de dictado del curso, la organización dada a los contenidos, las propuestas pedagógicas, los contenidos teóricos y los materiales de lectura y audiovisuales propuestos.

Compartimos esta experiencia con el objetivo de aportar a la reflexión y construcción de estrategias para la construcción de sociedades más igualitarias, señalando la importancia del rol de la Universidad en el diseño e implementación de políticas vinculadas a la prevención y erradicación de las violencias.

Asimismo consideramos que el conocimiento sobre la percepción de quienes transitan estos espacios es fundamental para el fortalecimiento y desarrollo de trayectos formativos en la materia que permitan profundizar los avances logrados, actualizar los contenidos y construir una comunidad sin violencias.

\section{Bibliografía}

ARZAMENDIA, Laura.; BIAGETTI, Maria y FIGUEROA MACHADO, Lucas. (2017). Mujeres en la acción colectiva. Fenómeno del "Ni una menos". Villa María: Universidad Nacional de Villa María. Disponible en línea en: http://biblio.unvm.edu.ar/opac_css/doc_num.php?explnum_id=1431

CAROSIO, Alba (2009). "Feminismo latinoamericano: imperativo ético para la emancipación". En GIRÓN, Alicia (Coord). Género y glogabización. Buenos Aires : Consejo Latinoamericano de Ciencias Sociales - CLACSO

Revista Zona Franca- Centro de estudios interdisciplinario sobre las mujeres (CEIM)- Maestría poder y sociedad desde la problemática de género (MG), Rosario, Argentina. ISSN, 2545-6504 http://zonafranca.unr.edu.ar/index.php/ZonaFranca| Número 29 (2021). 
MOLTONI, Rocio (2018). Lo personal es política universitaria. Un análisis de las tramas feministas que originan y componen la Red Interuniversitaria por la igualdad de género y contra las violencias. Tesina de grado, Universidad de Rosario. Disponle en línea

en: https://rephip.unr.edu.ar/bitstream/handle/2133/11543/Tesina\%20\%20Lic.\%20Cien cia\%20Pol\%C3\%ADtica\%20-\%20MOLTONI.pdf?sequence=3\&isAllowed=y

RODIGOU, Maite, BLANES Paola y DOMINGUEZ, Alejandra. (2011). Trabajar en la Universidad: (Des) Igualdades de género por transformar. Universidad Nacional de Córdoba.

RODINO, Ana María (2014). Pensar la educación en derechos humanos como política pública. Revista de ciencias sociales, segunda época 129 № 25, otoño de 2014, pp. 129-139.

TORLUCCI, Sandra; VAZQUEZ LABA, Vanesa; PÉREZ TORT, Mailén (2019). La segunda reforma universitaria: políticas de género y transversalización en las universidades. REVCOM. Revista científica de la red de carreras de Comunicación Social, núm. 9, 2019 Universidad Nacional de La Plata, Argentina. Disponible en línea

en:

http://ruge.cin.edu.ar/attachments/article/34/ACFrOgBh34eT2KtBJBzfxCVQ5eSEiE BHEEaVt3n8GJliljvvulaKacwzebMb7PvjVe3x08KS7NSdmTEpycEoWnNF5lkkkzH

\section{IEIYazm4UqWI QqRvleNHIwzl2WQrvZNLTuJQWPVXaWgmh7X QsS.pdf}

Vázquez Laba, Vanesa, Palumbo, Mariana y FERNANDEZ, Carla (2016). ¿Cómo prevenir, sancionar y erradicar la violencia de género en las Universidades? Revista Ciencias Sociales. Facultad de Ciencias Sociales, Universidad de Buenos Aires, 106-115. Disponible en línea en: https://ri.conicet.gov.ar/handle/11336/116307

Revista Zona Franca- Centro de estudios interdisciplinario sobre las mujeres (CEIM)- Maestría poder y sociedad desde la problemática de género (MG), Rosario, Argentina. ISSN, 2545-6504 http://zonafranca.unr.edu.ar/index.php/ZonaFrancal Número 29 (2021). 